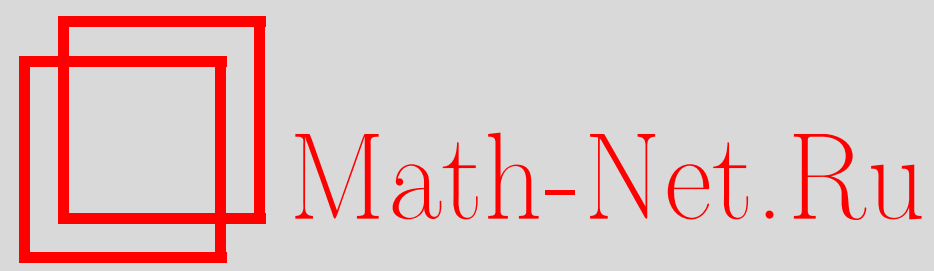

Н. А. Гусев, Слабая и сильная сходимость решений линеаризованных уравнений слабосжимаемой жидкости, Вестн. Сам. гос. техн. ун-та. Сер. Физ.-мат. науки, 2011, выпуск 1(), 47-52

DOI: https://doi.org/10.14498/vsgtu901

Использование Общероссийского математического портала Math-Net.Ru подразумевает, что вы прочитали и согласны с пользовательским соглашением http://www.mathnet.ru/rus/agreement

Параметры загрузки:

IP : 54.224 .187 .69

26 апреля 2023 г., 13:42:06 
УДК 532.51+517.956.6+517.972.5

\section{СЛАБАЯ И СИЛЬНАЯ СХОДИМОСТЬ РЕШЕНИЙ ЛИНЕАРИЗОВАННЫХ УРАВНЕНИЙ СЛАБОСЖИМАЕМОЙ ЖИДКОСТИ}

\section{Н. А. Гусев}

Московский физико-технический институт (государственный университет), 141700, Московская обл., Долгопрудный, Институтский переулок, 9.

Email: n.a.gusev@gmail.com

Рассматривается начально-краевая задача для линеаризованных уравнений вязкой баротропной слабосжимаемой жидкости в ограниченной области. Изучается поведение решений этой задачи при стремлении фактора сжсммемости $\kappa$ нулю. Приводятся достаточные условия слабой и сильной сходимости этих решений к решению соответствующей задачи для несжимаемой жидкости.

Ключевые слова: линеаризованные уравнения сжимаемой жидкости, фактор сжимаемости, слабосжимаемая жидкость.

Введение. Для математического описания механики жидкостей, как правило, используется модель несжимаемой жидкости. Эта модель является идеализацией, так как любая реальная жидкость, существующая в природе, является слабосжимаемой. В связи с этим возникает задача об исследовании свойств решений уравнений слабосжимаемой жидкости, в частности, сходимости этих решений к решению уравнений несжимаемой жидкости.

Поведение решений уравнений сжимаемой жидкости при стремлении числа Маха к нулю (что следует из сходимости сжимаемости к нулю) исследовалось в работах [1-4]. В частности, было доказано (см. [1,4]) существование последовательности слабых решений начально-краевой задачи для этих уравнений, для которой поле скорости сходится слабо к полю скорости несжимаемой жидкости. Однако с физической точки зрения сильная сходимость поля скорости (а также сходимость давления) представляет значительный интерес, поскольку в случае сильной сходимости несжимаемая жидкость точнее аппроксимирует сжимаемую.

Сильная сходимость поля скорости была установлена для искусственной системы уравнений сжимаемой жидкости, используемой при численном решении уравнений несжимаемой жидкости методом искусственной сжимаемости (см., например, [5, III, §8]). Также была установлена *-слабая сходимость градиента давления. Сильная сходимость давления не исследовалась.

При некоторых условиях сходимость решений уравнений сжимаемой жидкости не может быть сильной. Так, в [6] было получено необходимое условие сильной сходимости классических решений уравнений сжимаемой жидкости при стремлении фактора сжимаемости к нулю. Это условие представляет собой ограничение на начальное условие для уравнений несжимаемой жидкости и, вообще говоря, не выполняется.

В данной работе рассматриваются слабые решения линеаризованных уравнений слабосжимаемой жидкости. Это обусловлено тем, что на данный момент для полных уравнений сжимаемой жидкости установлено существова-

Николай Анатольевич Гусев, аспирант, каф. высшей математики. 
ние слабых решений, а вопрос о существовании сильных решений открыт [2]. Кроме того, линеаризация уравнений сжимаемой позволяет сосредоточиться на исследовании влияния сжимаемости на решение. Стоит также отметить, что линеаризованные уравнения сжимаемой жидкости представляют и самостоятельный интерес (например, в [7] исследовались спектральные свойства оператора, соответствующего стационарным линеаризованным уравнениям сжимаемой жидкости).

Линеаризованные уравнения движения сжимаемой жидкости рассматривались в $[8,9]$. В [9] была получена априорная оценка для сильного обобщённого решения начально-краевой задачи для этих уравнений в ограниченной области $D \subset \mathbb{R}^{3}$. Существование сильного обобщённого решения задачи Коши в $\mathbb{R}^{3}$ для этих уравнений было установлено в [8]. Вопросы существования и единственности слабых решений, по-видимому, не рассматривались.

В данной работе приводятся достаточные условия существования и единственности слабых решений начально-краевой задачи для линеаризованных уравнений слабосжимаемой жидкости. Исследуется сходимость этих решений при стремлении сжимаемости к нулю. Основные результаты об этой сходимости состоят в следующем:

- в общем случае поле скорости сходится слабо;

- если начальное условие для поля скорости соленоидально, то поле скорости сходится сильно и поле давления сходится слабо;

- если, кроме того, начальное условие для давления совпадает со значением давления в несжимаемой жидкости в начальный момент времени, то сходимость поля давления является сильной.

1. Обозначения. Рассмотрим ограниченную область $D \subset \mathbb{R}^{d}(d \in \mathbb{N}, d \geqslant 2)$ с кусочно-гладкой границей $\partial D$. Пусть $T>0, Q_{T}=D \times(0, T)$.

В данной работе используются стандартные обозначения для пространств Лебега, Соболева, Лебега-Бохнера и т. п. (см., например, [10]).

Как известно, пространство $H_{0}^{1}(D)^{k}(k \in \mathbb{N})$ со скалярным произведением

$$
((\boldsymbol{u}, \boldsymbol{v}))=\int_{D}(\nabla \otimes \boldsymbol{u}):(\nabla \otimes \boldsymbol{v}) d x \equiv \int_{D}\left(\partial_{i} u_{k}\right)\left(\partial_{i} v_{k}\right) d x
$$

является гильбертовым (по повторяющимся индексам ведётся суммирование.) Здесь $\{\boldsymbol{u}, \boldsymbol{v}\} \subset H_{0}^{1}(D)^{k}$ - вектор-функции.

С помощью $(\cdot, \cdot)$ в зависимости от контекста обозначается стандартное скалярное произведение в $\mathbb{R}^{k}$ либо скалярное произведение в $L^{2}(D)^{k}$, т. е. $(\boldsymbol{u}, \boldsymbol{v})=\int_{D} u_{i} v_{i} d x$

Пусть $X$ - банахово пространство и $\left(x_{n}\right) \subset X, x \in X$. Введём обозначения:

$x_{n} \rightarrow x \quad \Leftrightarrow \quad\left(x_{n}\right)$ сходится к $x$ слабо;

$x_{n} \rightarrow x \quad \Leftrightarrow \quad\left(x_{n}\right)$ сходится к $x *$-слабо.

Пусть $\dot{J}(D)$ - пространство бесконечно дифференцируемых финитных соленоидальных векторных полей. Пусть $J(D)$ и $V(D)$ - замыкания $\dot{J}(D)$ по норме $L^{2}(D)^{d}$ и $H_{0}^{1}(D)^{d}$. Обозначим через $P_{J}$ ортогональный проектор $L^{2}(D)^{d}$ на $J(D)$, часто называемый проектором Лерэ-Гельмгольиа. 
2. Линеаризованные уравнения слабосжимаемой жидкости. Рассмотрим вязкую баротропную жидкость с уравнением состояния $\rho=F(p)$, где $\rho$ и $p-$ плотность и давление соответственно. Линеаризуем это уравнение в окрестности некоторого значения давления $p_{\mathrm{ref}}$ :

$$
\rho=F(p) \approx F\left(p_{\text {ref }}\right)+F^{\prime}\left(p_{\text {ref }}\right)\left(p-p_{\text {ref }}\right) .
$$

Для краткости будем считать, что $p_{\text {ref }}=0$ и обозначим $F(0)=\rho_{0}>0$, $F^{\prime}(0)=\alpha$. С физической точки зрения $1 / \alpha=\partial p / \partial \rho=c^{2}>0$, где $c$ - скорость звука. Итак, линеаризованное уравнение состояния имеет вид $\rho=\rho_{0}+\alpha p$.

Рассмотрим систему уравнений

$$
\rho_{t}+\rho_{0} \operatorname{div} \boldsymbol{u}=0, \quad \rho_{0} \boldsymbol{u}_{t}+\nabla p=\mu \Delta \boldsymbol{u}+\eta \nabla \operatorname{div} \boldsymbol{u}+\rho \boldsymbol{f}, \quad \rho=\rho_{0}+\alpha p .
$$

Здесь $\rho=\rho(x, t)(\rho(x, t) \in \mathbb{R}), x \in D \subset(0, T), t \in(0, T) \subset \mathbb{R}, p=p(x, t)$ $(p(x, t) \in \mathbb{R}), \boldsymbol{u}=\boldsymbol{u}(x, t)$ - поле скорости $\left(\boldsymbol{u}(x, t) \in \mathbb{R}^{d}\right), \boldsymbol{f}=\boldsymbol{f}(x, t)$ - внешняя массовая сила.

Коэффициенты вязкости $\mu>0$ и $\eta \geqslant \mu / 3$ предполагаются постоянными. Число $\alpha>0$ называется фактором сжимаемости [6].

Уравнения (1) получаются линеаризацией уравнений сжимаемой жидкости вблизи некоторого решения уравнений несжимаемой жидкости. Возникающие при этом конвективные члены $(\boldsymbol{v}, \nabla) \alpha p$ и $(\boldsymbol{v}, \nabla) \boldsymbol{u}$ для простоты опущены.

Поставим для уравнений (1) следующие начальные и краевые условия:

$$
\left.\boldsymbol{u}\right|_{\partial D}=0,\left.\quad \boldsymbol{u}\right|_{t=0}=\boldsymbol{u}_{0},\left.\quad p\right|_{t=0}=p_{0} .
$$

Решение задачи (1), (2) зависит от фактора сжимаемости $\alpha$ :

$$
\{\boldsymbol{u}, p\}=\left\{\boldsymbol{u}_{\alpha}, p_{\alpha}\right\} .
$$

Основной целью данной работы является изучение сходимости этих решений при $\alpha \rightarrow 0$. Однако перед исследованием этого предельного перехода следует дать определения слабого решения задачи (1), (2) и слабого решения соответствующей задачи для несжимаемой жидкости.

3. Существование и единственность слабых решений. Предположим что $\boldsymbol{f} \in L^{\infty}\left(Q_{T}\right)^{d}, \boldsymbol{u}_{0} \in L^{2}(D)^{d}, p_{0} \in L^{2}(D)$.

ОПредЕЛЕние 1 . Пара $\{\boldsymbol{u}, p\} \in L^{2}\left(0, T ; H_{0}^{1}(D)^{d}\right) \times L^{2}\left(0, T ; L^{2}(D)\right)$ называется слабым решением задачи (1), (2), если для $\forall \varphi \in C_{0}^{\infty}([0, T)), \forall \boldsymbol{v} \in H_{0}^{1}(D)^{d}$ и $\forall r \in L^{2}(D)$

$$
\begin{aligned}
& -\int_{0}^{T}(\alpha p, r) \varphi_{t} d t-\left(\alpha p_{0}, r\right) \varphi(0)+\int_{0}^{T}\left(\rho_{0} \operatorname{div} \boldsymbol{u}, r\right) \varphi d t=0 \\
& -\int_{0}^{T} \rho_{0}(\boldsymbol{u}, \boldsymbol{v}) \varphi_{t} d t-\rho_{0}\left(\boldsymbol{u}_{0}, \boldsymbol{v}\right) \varphi(0)-\int_{0}^{T}(p, \operatorname{div} \boldsymbol{v}) \varphi d t= \\
& =-\mu \int_{0}^{T}((\boldsymbol{u}, \boldsymbol{v})) \varphi d t-\eta \int_{0}^{T}(\operatorname{div} \boldsymbol{u}, \operatorname{div} \boldsymbol{v}) \varphi d t+\int_{0}^{T}\left(\left(\rho_{0}+\alpha p\right) \boldsymbol{f}, \boldsymbol{v}\right) \varphi d t
\end{aligned}
$$


Теорема 1. Задача (1), (2) имеет единственное слабое решение $\{\boldsymbol{u}, p\}$.

До ка з а те ль с т в о проводится по методу Фаэдо-Галёркина.

4. Уравнения несжимаемой жидкости. При $\alpha=0$ уравнения (2) формально переходят в нестационарные уравнения Стокса для несжимаемой жидкости

$$
\operatorname{div} \boldsymbol{v}=0, \quad \rho_{0} \boldsymbol{v}_{t}+\nabla q=\mu \Delta \boldsymbol{v}+\rho_{0} \boldsymbol{f} .
$$

Поставим для уравнений (3) следующие начальные и краевые условия:

$$
\left.\boldsymbol{v}\right|_{\partial D}=0,\left.\quad \boldsymbol{v}\right|_{t=0}=\boldsymbol{v}_{0} .
$$

ОПРедЕление 2. Пара $\{\boldsymbol{v}, q\} \in L^{2}(0, T ; V(D)) \times \mathcal{D}^{\prime}\left(Q_{T}\right)$ называется слабым решением задачи (3), (4), если (3) выполняется в $\mathcal{D}^{\prime}\left(Q_{T}\right)$ и для $\forall \varphi \in$ $C_{0}^{\infty}([0, T)), \forall \boldsymbol{v} \in V(D)$

$$
-\int_{0}^{T} \rho_{0}(\boldsymbol{v}, \boldsymbol{v}) \varphi_{t} d t-\rho_{0}\left(\boldsymbol{v}_{0}, \boldsymbol{v}\right) \varphi(0)=-\mu \int_{0}^{T}((\boldsymbol{v}, \boldsymbol{v})) \varphi d t+\int_{0}^{T}\left(\rho_{0} \boldsymbol{f}, \boldsymbol{v}\right) \varphi d t
$$

Достаточные условия существования слабого решения задачи (3), (4) приведены, например, в [5]. Регулярность решений задачи (3), (4) изучалась В. А. Солонниковым (см., например, [11]).

5. Сходимость к решению уравнений несжимаемой жидкости. Пусть $\left\{\boldsymbol{u}_{\alpha}, p_{\alpha}\right\}_{0<\alpha<1}-$ множество решений задачи (1), (2) (существование и единственность которых следует из теоремы 1$)$. Пусть $\{\boldsymbol{v}, q\}$ - слабое решение задачи (3), (4) с начальным условием $\boldsymbol{v}_{0}=P_{J} \boldsymbol{u}_{0}$.

Teopema 2. При $\alpha \rightarrow 0$

$$
\begin{gathered}
\boldsymbol{u}_{\alpha} \rightarrow \boldsymbol{v} \quad \text { в } L^{2}\left(0, T ; H_{0}^{1}(D)^{d}\right), \quad \boldsymbol{u}_{\alpha} \rightarrow \boldsymbol{v} \quad \text { в } L^{\infty}\left(0, T ; L^{2}(D)^{d}\right), \\
\nabla p_{\alpha} \rightarrow \nabla q \quad \text { в } H^{-1}\left(Q_{T}\right) .
\end{gathered}
$$

Доказ ательств в проводится с помощью оценок слабых решений задачи (1), (2), теоремы Алаоглу-Бурбаки и единственности решения задачи (3), (4).

Теорема 2 согласуется с результатами, полученными в $[1,4]$. Однако с помощью рассуждений, использованных в методе искусственной сжимаемости [5], можно усилить топологию сходимости (5), (6)

Tеорема 3. Если $\boldsymbol{u}_{0} \in J(D)$, то при $\alpha \rightarrow 0$

$$
\boldsymbol{u}_{\alpha} \rightarrow \boldsymbol{v} \quad \text { в } L^{2}\left(0, T ; H_{0}^{1}(D)^{d}\right), \quad \nabla p_{\alpha} \rightarrow \nabla q \quad \text { в } H^{-1}\left(Q_{T}\right) .
$$

Важное отличие уравнений сжимаемой жидкости от уравнений несжимаемой жидкости состоит в том, что только для первых выполняется закон сохранения массы. А именно, пусть

$$
M(t)=\int_{D} \rho d x=\int_{D}(1+\alpha p) d x .
$$


Тогда из (1) получаем $d M / d t=0$. Следовательно, $\int_{D} p(t) d x$ не зависит от $t$. Для несжимаемой жидкости $\int_{D} q(t) d x$ в общем случае зависит от $t$, так как давление $q$ определено с точностью до аддитивной функции времени. Однако для произвольного числа $A \in \mathbb{R}$ эту функцию можно определить так, что $\int_{D} q(t) d x=A$ для п. в. $t \in[0, T]$.

Teopema 4. Пyсmъ $\boldsymbol{u}_{0} \in J(D) u$

$$
\begin{gathered}
\boldsymbol{v} \in \widetilde{W}^{1,2}\left(0, T ; H_{0}^{1}(D)^{d}\right), \quad q \in W^{1,2}\left(0, T ; L^{2}(D)\right), \\
\int_{D} q(t) d x=\int_{D} p_{0} d x \quad \text { для n. в. } t \in[0, T] .
\end{gathered}
$$

Тогда $p_{\alpha} \rightarrow q$ в $L^{\infty}\left(0, T ; L^{2}(D)\right), \alpha \rightarrow 0$.

До каз а тель ст в о проводится с помощью оценки разности слабых решений $\left\{\boldsymbol{u}_{\alpha}-\boldsymbol{v}, p_{\alpha}-q\right\}$, удовлетворяющей неоднородной задаче (1), (2).

Сильная сходимость давления в общем случае невозможна, поскольку

$$
\left\|p_{\alpha}-q\right\|_{L^{\infty}\left(0, T ; L^{2}(D)\right)} \geqslant\left\|p_{0}-q(0)\right\|_{L^{2}(D)} .
$$

Теорема 5. Пусть выполнены все условия теоремы 4 и область $D$ является звёздной по отношению к некоторому шару. Если, кроме того,

$$
q \in W^{2,2}\left(0, T ; L^{2}(D)\right) \quad u \quad p_{0}=q(0),
$$

mo

$$
p_{\alpha} \rightarrow q \quad \text { в } \quad L^{\infty}\left(0, T ; L^{2}(D)\right), \quad \alpha \rightarrow 0 .
$$

Доказательство этой теоремы подобно доказательству теоремы 4, однако к $q_{t}$ дополнительно применяется оператор Боговского [12]).

Работа выполнена при поддержке ФЦП «Научные и научно-педагогические кадры инновационной России» (гос. контракты П532 и П938) и РФФИ (проект № 09-01-12157-офи-м).

\section{БИБЛИОГРАФИЧЕСКИЙ СПИСОК}

1. Feireisl E., Novotny A. The low mach number limit for the full Navier-Stokes-Fourier system // Arch. Ration. Mech. Anal., 2007. no. 186. Pp. 77-107.

2. Feireisl E. Dynamics of viscous compressible fluids / Oxford Lecture Series in Mathematics and its Applications. Vol. 26. Oxford: Oxford Univ. Press, 2004. 212 pp.

3. Feireisl E., Novotný A. The Oberbeck-Boussinesq approximation as a singular limit of the full Navier-Stokes-Fourier system // J. Math. Fluid Mech., 2009. Vol. 11, no. 2. Pp. 274-302.

4. Lions P.-L., Masmoudi N. Incompressible limit for a viscous compressible fluid// J. Math. Pures Appl., IX. Sér., 1998. Vol.77, no.6. Pp. 585-627.

5. Temam R. Navier-Stokes equations. Theory and numerical analysis/ Studies in Mathematics and its Applications. Vol. 2. Amsterdam - New York - Oxford: North-Holland, 1979. $519 \mathrm{pp}$. 
6. Шифрин Э.Г. Условие непрерывной зависимости от сжимаемости нестационарных течений вязких мало сжимаемых жидкостей // Докл. РАН, 1999. Т. 365, № 2. С. 197200; англ. пер.: Shifrin E. G. Unstable Flows in Viscous Slightly Compressible Fluids. The Condition of Continuous Dependence on Compressibility // Dokl. Phys., 1999. Vol. 44, no. 3. Pp. 189-192.

7. Прибыль М. А. Спектральный анализ линеаризованных стационарных уравнений вязкой сжимаемой жидкости, заданных в $\mathbb{R}^{3}$ с периодическими краевыми условиями // Алгебра и анализ, 2008. Т. 20, № 2. С. 149-177; англ. пер.: Pribyl' M. A. Spectral analysis of linearized stationary equations of viscous compressible fluid in $\mathbb{R}^{3}$, with periodic boundary conditions // St. Petersburg Math. J., 2009. Vol.20, no. 2. Pp. 267-288.

8. Ikehata R., Koboyashi T., Matsuyama T. Remark on the $L_{2}$ estimates of the density for the compressible Navier-Stokes flow in $\mathbb{R}^{3} / /$ Nonlinear Anal., Theory Methods Appl., 2001. Vol. 47, no. 4. Pp. 2519-2526.

9. Mucha P.B., Zajaczkowski W.M. On a $L_{p}$-estimate for the linearized compressible NavierStokes equations with the Dirichlet boundary conditions// J. Differ. Equations, 2002. Vol. 186, no. 2. Pp. 377-393.

10. Gasiński L., Papageorgiou N.S. Nonlinear Analysis / Series in Mathematical Analysis and Applications. Vol. 9. Boca Raton, FL: Chapman \& Hall/CRC, 2006. 971 pp.

11. Ладъженская O. А. Математические вопросы динамики вязкой несжимаемой жидкости. М.: Наука, 1970. 288 с.; англ. пер.: Ladyzhenskaya O. A. The boundary value problems of mathematical physics / Applied Mathematical Sciences. Vol.49. Berlin etc.: SpringerVerlag, 1985. 322 pp.

12. Боговский M. E. Решение первой краевой задачи для уравнения неразрывности несжимаемой среды // ДАН СCCP, 1979. Т. 248, № 5. С. 1037-1040. [Bogovskiy M. E. Solution of the first boundary value problem for an equation of continuity of an incompressible medium // DAN SSSR, 1979. Vol. 248, no. 5. Pp. 1037-1040].

Поступила в редакцию $21 / \mathrm{XII} / 2010$;

в окончательном варианте - 16/III/2011.

MSC: 35D30, 76N10

\section{WEAK AND STRONG CONVERGENCE OF SOLUTIONS TO LINEARIZED EQUATIONS OF LOW COMPRESSIBLE FLUID}

\section{N. A. Gusev}

Moscow Institute of Physics and Technology,

9, Inststitutskiy per., Dolgoprudniy, Moskovskaya obl., 141700, Russia.

Email: n.a.gusev@gmail.com

Initial-boundary value problem for linearized equations of viscous barotropic low compressible fluid in a bounded domain is considered. Convergence of solutions of this problem at withincompressible limit approaching to zero is studied. Sufficient conditions for the weak and strong convergence of this problem for uncompressible liquid are given.

Key words: linearized equations of compressible fluid, compressibility factor, low compressible fluid.

Original article submitted $21 / \mathrm{XII} / 2010$; revision submitted 16/III/2011.

Nikolay A. Gusev, Postgraduate Student, Dept. of Higher Mathematics. 